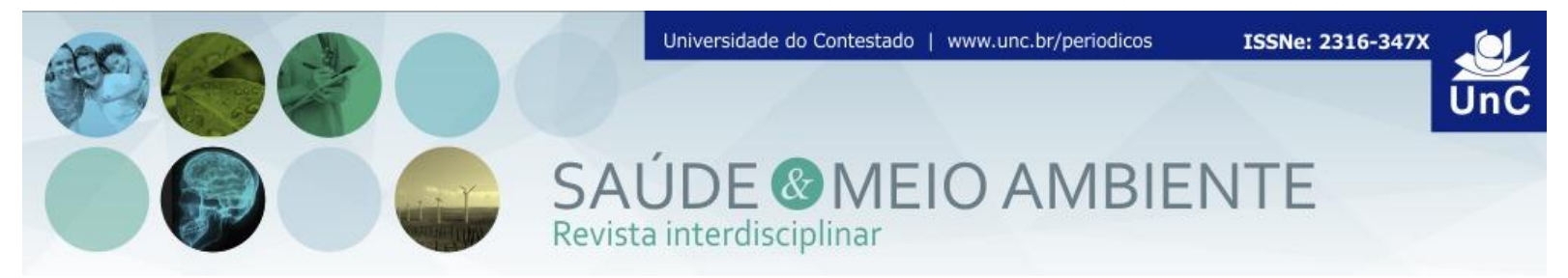

\title{
ÁCARO PLUMÍCOLA NYMPHICILICHUS PEREZAE EM CALOPSITAS
}

\author{
Daniela Pedrassani ${ }^{1}$ \\ Daniele de Cassia Karvat \\ Jéssica Thays Ebertz ${ }^{3}$
}

\begin{abstract}
RESUMO
As aves silvestres estão se tornando cada vez mais populares como animais de companhia, porém as suas penas constituem um ambiente propício à sobrevivência e reprodução de uma grande variedade de ácaros. No Brasil, os estudos sobre ácaros de penas ainda são escassos. Esses ácaros plumícolas são ectoparasitas obrigatórios, e são transmitidos por meio do contato direto entre aves, principalmente da mesma espécie, já que suas penas favorecem um habitat adequado para a sobrevivência dos ácaros. Esse trabalho tem o objetivo de relatar casos clínicos da ocorrência do ácaro plumícola Nymphicilichus perezae em duas calopsitas (Nymphicus hollandicus) atendidas em uma universidade no município de Canoinhas, Santa Catarina. As aves apresentavam prurido intenso, perda de penas acentuada e autobicamento. Após avaliadas as amostras de penas em microscópio estereoscópico, o ácaro plumícola $N$. perezae foi identificado. O tratamento instituído com permetrina foi eficaz na eliminação dos ácaros, e houve remissão completa dos sinais clínicos. Esse ácaro foi recentemente descrito e são poucos os relatos de sua presença ocasionando sinais clínicos em calopsitas no Brasil e no mundo.
\end{abstract}

Palavras-Chave: Ácaro. Astigmata. Nymphicus hollandicus. Nymphicilichus perezae.

\section{FEATHER MITE NYMPHICILICHUS PEREZAE IN COCKATIELS}

\begin{abstract}
Wild birds are becoming increasingly popular as pets, however their sentences constitute an environment conducive to the survival and reproduction of a wide variety of mites. In Brazil, there are few studies about feather mite. These feather mites are obligatory ectoparasites and are transmitted through direct contact between birds, mainly of the same species, since its feathers favor a suitable habitat for the survival of the mites. This work aims to report clinical cases of the occurrence of the feather mite Nymphicilichus perezae in two cockatiels (Nymphicus hollandicus)

\footnotetext{
${ }^{1}$ Graduada em Medicina Veterinária Universidade Federal do Paraná (UFPR). Mestre em Ciências Veterinárias Universidade Federal do Paraná (UFPR). Doutorado Medicina Veterinária Preventiva Universidade Estadual Paulista (UNESP). Professora do Depto de Medicina Veterinária. Universidade do Contestado (UnC) Canoinhas. Santa Catarina. Brasil. E-mail: daniela@unc.br

${ }^{2}$ Acadêmica do curso de Medicina Veterinária da UnC Campus Canoinhas. Canoinhas, Santa Catarina. Brasil. E-mail: dani.ellyn 4@hotmail.com

${ }^{3}$ Acadêmica do curso de Medicina Veterinária da UnC Campus Canoinhas. Canoinhas, Santa Catarina. Brasil. E-mail: ebertzthaysjessi@hotmail.com
} 
who have received veterinary care in a University in the city of Canoinhas, Santa Catarina State. The birds had intense itching, loss of feathers and feather-picking behavior. After evaluated samples of feathers, the $N$. perezae mite has been identified. The treatment with permethrin was effective in eliminating of the mites, and there was complete remission of clinical signs. This mite has recently been described and there are few reports of his presence in cockatiels in Brazil and in the world causing clinical signs.

Keywords: Mite. Astigmata. Nymphicus hollandicus. Nymphicilichus perezae.

\section{INTRODUÇÃO}

Os animais selvagens, e principalmente as aves, estão se tornando cada vez mais populares como animais de companhia, devido a sua beleza em cantos e cores (GONDIM et al., 2006). Desse modo, a clínica médica e cirúrgica de animais selvagens vem adquirindo crescente importância na prática veterinária devido à preocupação com a saúde e bem-estar desses animais. A necessidade dos profissionais da área de veterinária em instruírem-se a respeito de tais animais se torna, assim, crucial (SCHULTE; RUPLEY, 2004).

A calopsita (Nymphicus hollandicus Kerr, 1792) é um psitacídeo e têm sua origem na Austrália, foi introduzida no Brasil de forma organizada a partir da década de 1970, e atualmente é criada como ave de estimação (UNIVERSO AVES, 2012). É a segunda espécie de psitaciforme mais criada no mundo, como ave de estimação, e em 1998, foi classificada pelo Instituto Brasileiro do Meio Ambiente e dos Recursos Humanos Renováveis (IBAMA), como pertencente a fauna doméstica (MAIA, 2009).

Embora o conhecimento sobre parasitas de aves de produção seja relativamente extenso, estudos sobre parasitas de aves silvestres ainda são restritos (ENOUT et al., 2009).

Roda e Farias (1999), afirmam que as aves silvestres abrigam uma fauna de ectoparasitas típica. De acordo com Gaud e Atyeo (1979), cada ordem da classe aves apresenta uma ácarofauna definida, o que demonstra uma íntima adaptação ao seu hospedeiro.

Os ectoparasitas podem afetar as aves diretamente, alimentando-se delas, ou indiretamente, pois podem servir como vetores de protozoários, bactérias e vírus (STORNL; ALVES; VALIM, 2005).

Os ácaros que parasitam aves incluem-se em três grandes grupos: Mesostigmata, Prostigmata e Astigmata (KNEE; PROCTOR; GALLOWAY, 2008). Os ácaros plumícolas compõem o grupo mais numeroso de artrópodes, os quais estão associados as aves (KANEGAE et al., 2008). Pertencem a subordem Astigmata e as superfamílias Analgoidea, Pterolichoidea e Freyanoidea (GAUD; ATYEO 1996), 
sendo a primeira apenas encontrada em aves Passeriformes (KANEGAE et al., 2008).

Atualmente, no Brasil, existem poucos estudos sobre a ecologia e identificação de ácaros plumícolas. Os primeiros estudos sobre ácaros no país apresentaram um enfoque taxonômico e foram publicados por Hebert F. Berla, no meio século passado (LYRA NEVES et al., 2003; KANEGAE et al., 2008).

O ciclo de vida dos ácaros plumícolas desenvolve-se sob um único hospedeiro, e os estágios que compõem sua biologia são: ovo, larva, protoninfa, tritoninfa e adultos (VON MATTER et al., 2010).

De acordo com Proctor e Owens (2000), os ácaros alimentam-se principalmente de óleo proveniente da glândula uropigial, de descamações, pólen e fungos que aderem às barbas das penas. Partículas de penas são raramente observadas no intestino desses ácaros (PROCTOR; OWENS, 2000).

Com relação a ordem Psittaciformes tem sido relatado a presença de ácaros plumícolas, de diversas famílias: Apionacaridae, Dermoglyphidae, Proctophyllodidae, Psoroptoididae, Ptyssalgidae, Xolalgidae, Ascouracaridae, Falculiferidae e Pterolichidae (GAUD; ATYEO, 1996).

Os ácaros de penas da família Pterolichidae associados aos Psittaciformes estão divididos em três grupos genéricos: Protolichus, Rhytidelasma e Psittophagus (GAUD; ATYEO, 1996).

No Brasil Albuquerque et al. (2012) verificaram para a espécie de Psittaciforme Nymphicus hollandicus que as rêmiges primárias (direita e esquerda) foram as penas mais infestadas pelos ácaros Nymphicilichus perezae, Psittophagus sp. e Dubininia sp.

O gênero Nymphicilichus é considerado derivado grupo Psittophagus, e o que o difere das outras espécies do grupo é a ausência da seta $h 1$ e da solenídea $\sigma 1$ nos genus II e III, além de significativa redução do escudo histerossomal (MIRONOV et al., 2003). A espécie Nymphicilichus perezae foi descrita por Mironov e Galloway em 2002, em exemplares de calopsitas, provenientes da Nova Zelândia, e em 2003 Mironov et al. fizeram algumas correções em sua descrição original (MIRONOV et al., 2003).

O primeiro relato do ácaro $N$. perezae no Brasil, infestando exemplares de Nymphicus hollandicus foi em 2010, no Setor de Animais Selvagens no Hospital Veterinário da Universidade Federal Fluminense em Niterói, Rio de Janeiro. Neste estudo, das 177 amostras de penas analisadas, a espécie de ácaro mais prevalente foi $N$. perezae $(32,43 \%)$ (ALBUQUERQUE et al., 2012).

O objetivo deste trabalho é relatar a ocorrência do ácaro plumícola Nymphicilichus perezae em duas calopsitas, recebidas para atendimento veterinário na Universidade do Contestado. 
Maiores estudos sobre ácaros plumícolas são urgentes no Brasil pela necessidade de se conhecer o comportamento das populações de ácaros de penas de forma a esclarecer a relação ácaro de pena versus hospedeiro e entre as diversas espécies de ácaros plumícolas no mesmo hospedeiro.

\section{METODOLOGIA}

O presente estudo trata-se de um "relato de casos clínicos". Os relatos de casos são uma ferramenta de fundamental importância especialmente para os profissionais de saúde humana e animal, que se deparam diariamente com casos raros, difíceis, interessantes ou de evolução pouco comum, e que precisam ser informados a comunidade científica (ARAGÃO; TAVARES, 2009).

No relato clinico, apresenta-se os casos de duas calopsitas, fêmeas, adultas, que foram atendidas na Universidade do Contestado, no Setor de Animais Silvestres, apresentando escore corporal normal, irritação de pele, prurido intenso em todo o corpo, perda de penas acentuada e autobicamento. Na anamnese foi relatado que as aves têm contato direto com outras aves silvestres que adentram na área de varanda onde as aves permanecem durante parte do dia. $\mathrm{O}$ tutor ainda relatou que uma das aves foi adquirida em criadouro e que a outra foi encontrada na varanda de sua residência, onde permaneceu e, que foi por ele adotada.

No exame físico das aves, a plumagem e a pele foram cuidadosamente inspecionadas. $O$ tutor também trouxe as penas que foram perdidas pelas aves no dia do atendimento, essas foram preservadas em etanol $70 \mathrm{GL}$ e examinadas sob microscópio estereoscópico.

Inicialmente observou-se a presença de ácaros e sua disposição nas penas, e estes foram então, retirados com auxílio de estiletes e montados entre lâmina e lamínula com solução de Hoyer e observados em microscopia óptica para identificação.

Com base nas descrições de Mironov e Galloway (2002), Mironov et al. (2003) e Albuquerque et al. (2012), o ácaro identificado foi o ácaro plumícola Nymphicilichus perezae.

Concluídas as identificações o material analisado foi depositado na Coleção de Ectoparasitas do Laboratório de Parasitologia Veterinária da Universidade do Contestado, Campus Canoinhas.

O tratamento foi prescrito com o medicamento a base de Permetrina $0,25 \%{ }^{4}$ na forma de talco a ser pulverizado diretamente sobre o corpo das calopsitas e também sobre o piso da gaiola por três dias consecutivos, cuidando para que este ${ }^{4}$ Piolhaves®. PROVETS SIMÕES LABORATÓRIO LTDA. RUA PEREIRA DE ALMEIDA, 90 / 90 A PRAÇA DA BANDEIRA
20260-100 - RIO DE JANEIRO - RJ. 
não caia sobre a comida e a água, devido ao risco de intoxicação. Foi indicado repetir o tratamento depois de 15 dias.

\section{RESULTADOS E DISCUSSÃO}

Os ácaros estavam ocasionando danos ao bem-estar físico (lesões na pele e penas) e mental (autobicamento e comportamento agitado apresentado pelas aves e relatado pelo tutor) das calopsitas atendidas em uma relação tipicamente parasitária, com desequilíbrio na relação parasita-hospedeiro ocorrendo danos mais evidentes as aves.

Nas condições de cativeiro ocorre contato mais próximo e frequente entre as aves, o que permite a transmissão de ácaros de pena entre hospedeiros e, como as duas aves tem contato, uma pode ter infestado a outra.

Os ácaros plumícolas são descritos como parasitas por alguns autores (HARPER, 1999; PROCTOR, 2003) e por outros como tendo uma relação de ectosimbionte (mutualismo ou comensalismo) com as aves (ALBUQUERQUE et al., 2012; PROCTOR; OWENS, 2000). Arzua e Valim (2010) caracterizaram a relação entre aves e ácaros de pena como um tipo de parasitismo, que envolve completa dependência por parte desses ácaros em viver e se desenvolver no hospedeiro. Harper (1999) detectou uma correlação negativa entre a infestação por ácaros de penas e o escore corporal das aves, assim como um impacto negativo na plumagem das aves infestadas. Processos patológicos decorrentes da presença de ácaros plumícolas estão na maioria das vezes relacionados a aves mantidas em cativeiro, com pouca possibilidade ou impossibilitadas de voo, o que leva a um aumento populacional de ácaros, prurido e bicamento (PROCTOR, 2003).

A pele das aves se apresentava irritada e eritematosa, principalmente na região das asas devido ao prurido e ao autobicamento.

Os ácaros foram verificados com maior frequência nas penas das asas (Figura 1) similar ao local de parasitismo descrito por Albuquerque et al. (2012) para calopsitas atendidas no setor de animais selvagens do hospital veterinário da Universidade Federal Fluminense, porém sem observarem relação entre presença do ácaro e lesões nas bárbulas das penas. 
Figura 1 - Vista ventral da pena da calopsita apresentando ácaros (setas) entre as barbas e junto à região da raque (Aumento de 40x).

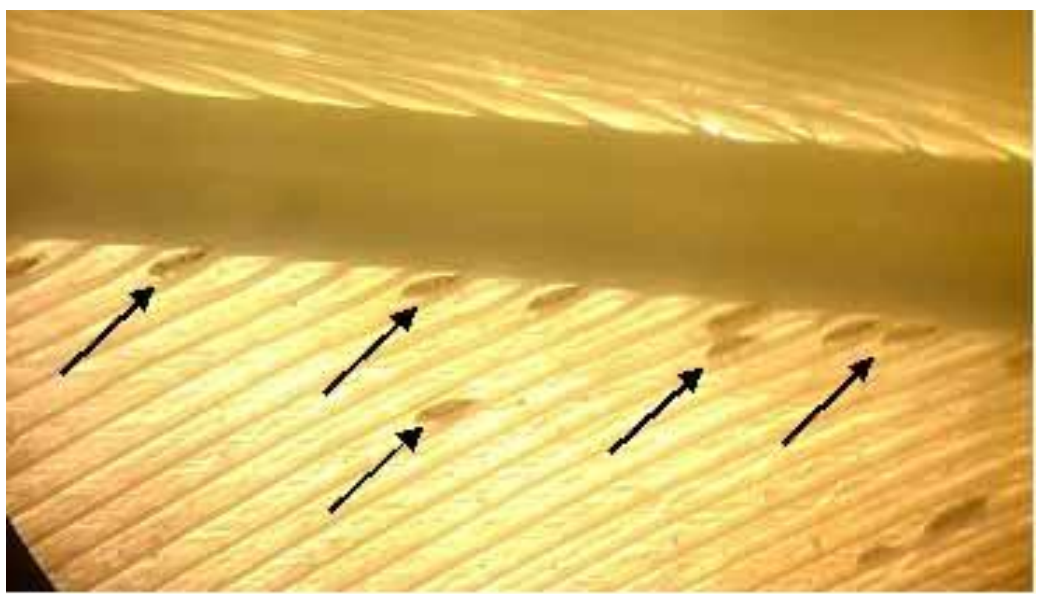

Fonte: Dados da Pesquisa (2015)

Pelas características morfológicas todos os ácaros das duas calopsitas foram identificados como Nymphicilichus perezae no qual as fêmeas apresentam, dorsalmente, um par de estruturas de formato ovalado, bem esclerotizadas, na região sejugal e outro na região opistossomal posterior (Figura 2). No macho, essas estruturas esclerotizadas estão presentes apenas na região opistossomal (Figura 3). As fêmeas medidas $(n=6)$ apresentaram um tamanho médio de $435 \mu \mathrm{m}$ de comprimento (idiossoma + gnatossoma) e $145 \mu \mathrm{m}$ de largura, medida essa similar a descrita por Mironov e Galloway (2002).

Figura 2 - Ácaro plumícola Nymphicilichus perezae (fêmea); aspecto dorsal: par de estruturas fortemente esclerotizadas em formato ovalado na região sejugal (seta vermelha) e na região posterior do opistossoma (seta preta) (Aumento de 100x).

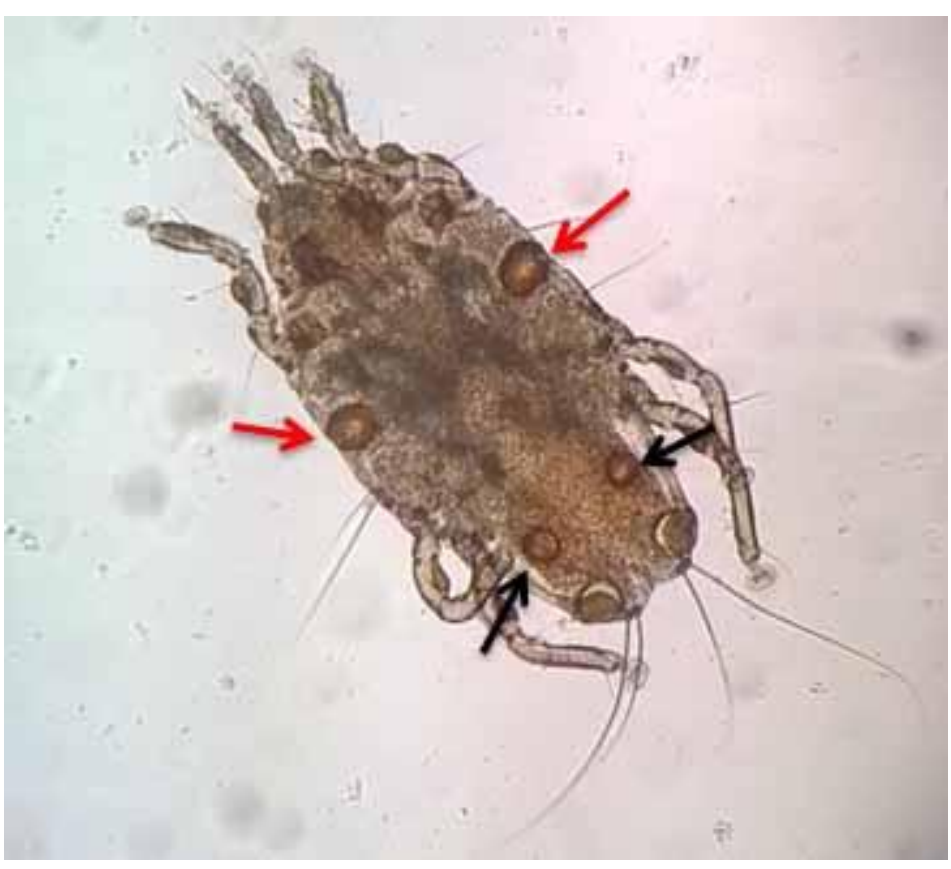

Fonte: Dados da Pesquisa (2015) 
Figura 3 - Ácaro plumícola Nymphicilichus perezae (macho); aspecto dorsal: par de estruturas fortemente esclerotizadas em formato ovalado ausente na região sejugal (seta vermelha) e presente na região posterior do opistossoma (seta preta) (Aumento de 46x).

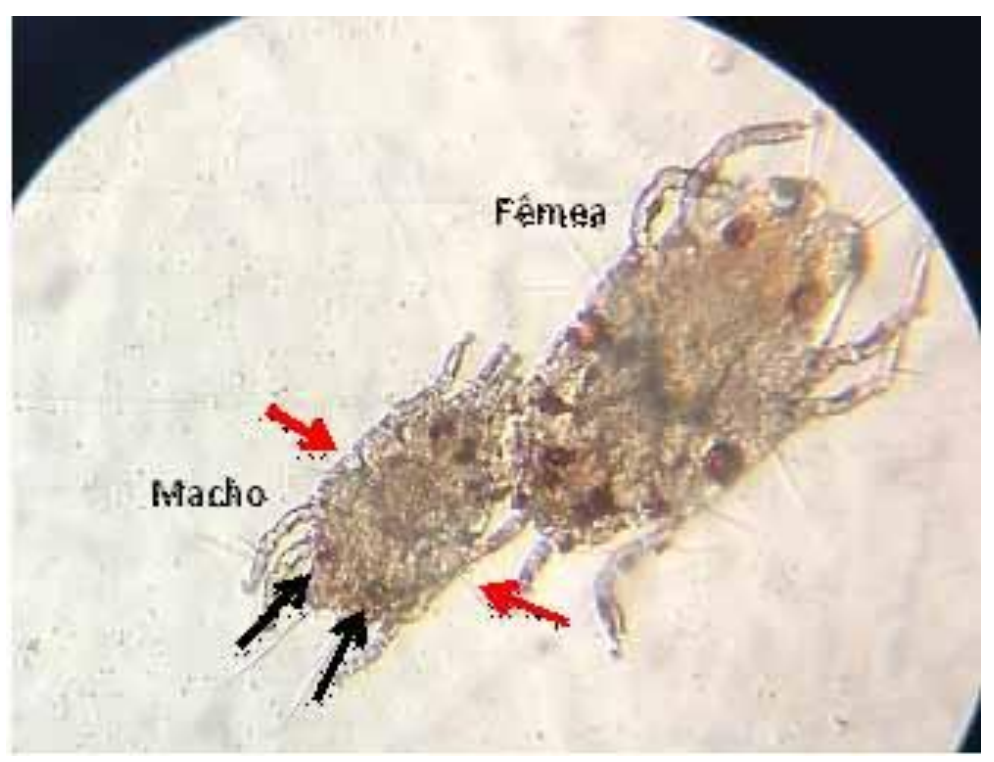

Fonte: Dados da Pesquisa (2015)

O controle de populações de ácaros de penas pode ser com produtos das classes Carbamatos e Piretróides (REZENDE et al., 2013). Pela baixa toxicidade optou-se por ectoparasiticida a base de Permetrina $0,25 \%$ na forma de talco, sendo realizadas no total cinco aplicações, sendo três com intervalo de cinco dias e duas com intervalo de 14 dias, seguindo assim as recomendações de administração do fabricante. As gaiolas das aves foram deixadas em ambiente arejado, limpas e também receberam aplicação do produto.

Houve boa resposta ao tratamento, bem como melhora do estado geral e diminuição significativa da irritação, prurido, perda de penas e do autobicamento. Após melhora do quadro clínico, foram recebidas mais amostras de penas, e realizado o mesmo procedimento a procura da presença do ácaro, mas o mesmo não foi encontrado, concluindo que o tratamento indicado foi eficiente.

\section{CONSIDERAÇÕES FINAIS}

A espécie de ácaro plumícola Nymphicilichus perezae, foi associada as alterações clínicas encontradas nas duas calopsitas, já que este foi o único ácaro presente nas amostras e pela remissão dos sinais clínicos após tratamento.

Os achados de danos ocasionados nas aves pela presença dos ectoparasitas nas penas reforçam a tese de relação interespecífica de parasitismo entre calopsitaácaro. Ainda, demonstra a necessidade de os médicos veterinários estarem sempre 
bem instruídos e atualizados em relação ao parasitismo em aves silvestres, tendo em vista que esses animais tendem a serem frequentadores mais assíduos em clínicas médicas veterinárias.

\section{REFERÊNCIAS}

ALBUQUERQUE, D. D. A. et al. The first identification of Nymphicilichus perezae Mironov and Galloway, 2002 in cockatiels in Brazil and the first record of Psittophagus sp. Gaud and Atyeo, 1996 and cf. Dubininia sp. Vassilev, 1958 in cockatiels (Nymphicus hollandicus Kerr, 1792). Parasitology International, v. 61, p. 572-578, 2012.

ARAGÃO, J.; TAVARES, M. Como Preparar Um Relato De Caso Clínico. Cadernos UniFOA, n. 9, abr., p. 59-62, 2009.

ARZUA, M., VALIM, M.P. Bases para o estudo qualitativo e quantitativo de ectoparasitos em aves In: STRAUBE, F.C; PIACENTINI, V. de Q. ACCORDI, L.A. Ornitologia e conservação; ciência aplicada, técnicas de pesquisa e levantamento. Rio de Janeiro: Technical Books, 2010. p. 347-366.

ENOUT, A.M.J., et al. Parasitismo por malófagos (Insecta) e ácaros (Acari) em Turdus leucomelas (Aves) nas estações reprodutiva e de muda de penas no parque Estadual do Rio Preto, Minas Gerais, Brasil. Zoologia, v.23, n.3, p.534-540, set.,2009.

GAUD, J. ; ATYEO, W.T. Nouvelles superfamilles pour les acariens astigmates parasites d'oiseaux. Acarologia, v.19, n.4, p. 678-685, 1977.

GAUD, J.; ATYEO, W.T. Feather mites of the world (Acarina, Astigmata): the supraspecific taxa. Annales, Sciences Zoologiques, Musee Royal de l'Afrique Centrale, v. 277, Parts 1 and 2, 1996.

GONDIM L.S.Q., GOMES D.M.; MAIA P.C.C. Casuística de aves selvagens atendidas de 2002 a 2004 na Escola de Medicina Veterinária da Universidade Federal da Bahia. Anais... Congresso Brasileiro de Zoologia, 26, Londrina, p. 86-87. 2006.

HARPER, D. G. C. Feather mites, pectoral muscle condition, wing length and plumage coloration of passerines. Animal Behaviour, v. 58, p. 553-562, 1999.

KANAGAE, M. F. et al. Ácaros plumícolas (Acari: Astigmata) em aves do Cerrado do Distrito Federal, Brasil. Biota Neotropica, v. 8, n. 1, p. 31-39, jan./mar. 2008.

KNEE, W.; PROCTOR, H.; GALLOWAY, T. Survey of nasal mites (Rhinonyssidae, Ereynetidae, and Turbinoptidae) associated with birds in Alberta and Manitoba, Canada. Canadian Entomologist, v. 140, p. 364-379, 2008. 
LYRA NEVES, R. M. et al. Ecological relationships between feather mites (Acari) and wild birds of Emberizidae (Aves) in a fragment of Atlantic Forest in northeastern Brazil. Revista Brasileira de Zoologia, Curitiba, v. 20, n.3, p.481- 485, 2003.

\section{MAIA, D.C.B.S.C. Leveduras Isoladas do Trato Gastrointestinal de Calopsitas} (Nymphicus hollandicus): Determinação da Microbiota e Análise Fenotípica. 98 p. Dissertação (Mestrado) - Universidade Estadual do Ceará, Fortaleza, CE, 2009.

MIRONOV, S.V. et al. A review of feather mites of the Psittophagus generic group (Astigmata, Pterolichidae) with descriptions of new taxa from parrots (Aves, Psittaciformes) of the Old World. Acta Parasitologica, v.48, n.4, 2003.

MIRONOV, S. V.; GALLOWAY, T. D. Nymphicilichus perezae gen. nov., sp. nov., a new feather mite (Astigmata: Pterolichidae) from the cockatiel, Nymphicus hollandicus (Psittaciformes: Cacatuidae), Journal of the Royal Society of New Zealand, v. 32, n. 1, p. 1-6, 2002.

PROCTOR, H. C.; OWENS, I. Mites and birds: diversity, parasitism and coevolution. Tree, v.15, n.9, p.358-364, 2000.

PROCTOR, H. C. Feather mites (Acari: Astigmata): ecology, behavior, and evolution. Annual Review of Entomology, v. 48, p. 185-209, 2003.

REZENDE, L. C.; CUNHA, L. M.; TEIXEIRA, C. M.; OLIVEIRA, P. R.; MARTINS, N. R. S. Mites affecting hen egg production - some considerations for Brazilian farms. Ciência Rural, v. 43, n. 7, p.1230-1237, 2013.

RODA, S.A.; FARIAS, A.M.I. Ácaros plumícolas em aves Passeriformes da Zona da Mata Norte de Pernambuco, Brasil. Revista Brasileira de Zoologia, Curitiba, v.16, n.3, p.879-886, 1999.

STORNL, A; ALVES, M. A. S.; VALIM, M.P. Ácaros de Penas e Carrapatos (Acari) Associados a Turdus albicollis Vieillot (Aves Muscicapidae) em Uma Area de Mata Atlântica da Ilha Grande, Rio de Janeiro, Brasil. Zoologia, v. 22, n. 2, p. 419-423, jun., 2005.

SCHULTE, M.S.; RUPLEY, A. E. Avian care and husbandry. Veterinary Clinics of North America: Exotic Animal Practice, v. 7, n. 2, p. 315-350, 2004.

UNIVERSO AVES. Calopsitas: Histórico, 2014. Disponível em: <http://www.omniverso.com/universoaves-calopsitas-historico.htm>. Acesso em: 09 ago. 2015.

VON MATTER, S. et al. Ornitologia e conservação: ciência aplicada, técnicas de pesquisa e levantamento. Rio de Janeiro: Techinical Books, 2010. Disponível em: <https://books.google.com.br>. Acesso em: 9 ago. 2015.

Artigo recebido em: 25/01/2016

Artigo aprovado em: 29/11/2016 